\title{
NonCOMMUTATIVE DIFFERENTIAL FORMS AND QUANTIZATION OF THE ODD SYMPLECTIC CATEGORY
}

\author{
Pavol Ševera \\ Dept. of Theoretical Physics \\ Comenius University \\ Bratislava, Slovakia \\ severa@sophia.dtp.fmph.uniba.sk
}

\begin{abstract}
There is a simple and natural quantization of differential forms on odd Poisson supermanifolds, given by the relation $[f, d g]=\{f, g\}$ for any two functions $f$ and $g$. We notice that this non-commutative differential algebra has a geometrical realization as a convolution algebra of the symplectic groupoid integrating the Poisson manifold.

This quantization is just a part of a quantization of the odd symplectic category (where objects are odd symplectic supermanifolds and morphisms are Lagrangian relations) in terms of $\mathbb{Z}_{2}$-graded chain complexes. It is a straightforward consequence of the theory of $\mathrm{BV}$ operator acting on semidensities, due to $\mathrm{H}$. Khudaverdian.

MSC2000: 58A10, 53D55, 53D05, 58A50

Keywords: differential forms, symplectic category, Batalin-Vilkovisky operator
\end{abstract}

\section{Introduction}

There is a well-known analogy (formulated by A. Weinstein [3]) between symplectic manifolds and vector spaces:

\begin{tabular}{r|l} 
symplectic manifold & vector space \\
Lagrangian submanifold & vector \\
product & tensor product \\
opposite sympl. form & dual space \\
Lagrangian relation & linear map
\end{tabular}

It is very fruitful, though it is just an analogy, i.e. there is no functor from the symplectic category to the category of vector spaces.

However, there is such a quantization functor, if we substitute symplectic manifolds with odd symplectic supermanifolds, and vector spaces with $\mathbb{Z}_{2}$-graded chain complexes, i.e. $\mathbb{Z}_{2^{-}}$ graded vector spaces $V=V_{0} \oplus V_{1}$ with a differential $D: V_{0} \rightarrow V_{1}, V_{1} \rightarrow V_{0}, D^{2}=0$. Namely, to an odd symplectic supermanifold $Y$ it associates the vector space of semidensities on $Y$, with $D$ the BV operator (due to $\mathrm{H}$. Khudaverdian). Before giving the details, let us look at the result of quantization of odd symplectic groupoids.

\section{Differential forms on odd Poisson supermanifolds}

Let $X$ be a supermanifold and $\Omega(X)$ the differential graded algebra of differential forms on $X$. By a filtered deformation of $\Omega(X)$ we mean a differential filtered algebra $A$ (i.e. a differential algebra $A$ with an increasing filtration $F^{0} \subset F^{1} \subset \ldots \subset A$ such that $d F^{i} \subset F^{i+1}$ ) with an 
isomorphism between $\Omega(X)$ and the differential graded algebra $G r A$ associated to $A$ (where $\left.(\operatorname{Gr} A)^{i}=F^{i} / F^{i-1}\right)$. We have the following simple theorem:

Theorem 1. There is a natural bijection between (isomorphism classes of) filtered deformations of $\Omega(X)$ and odd Poisson structures on $X$. It is given by the commutation relation

$$
[f, d g]=\{f, g\}
$$

where $f$ and $g$ are any functions on $X,[$,$] is the supercommutator in the deformed algebra$ and $\{$,$\} is the odd Poisson structure.$

Proof. Notice that $F^{0}=C^{\infty}(X)$. Since $\Omega(X)$ is generated (as an algebra) by functions on $X$ and by their differentials, the same is true for $A$ (as is easily proved by induction). Finally, for any $f, g \in F^{0}$ we have $[f, d g] \in F^{0}$ (because $\Omega(X)$ is graded commutative, i.e. there $[f, d g]=0$ ). The algebra $A$ is thus known (up to canonical isomorphism) if we know the function $\{f, g\}:=[f, d g]$ for every two functions $f$ and $g$. It is straightforward to verify that $\{f, g\}$ has to be an odd Poisson structure. The converse (i.e. given an odd Poisson structure, the formula (1) gives a fitered deformation of $\Omega(X)$ ) can also be easily verified directly, but we give a more conceptual proof of this fact, using quantization of odd symplectic groupoids, later.

If $\pi$ is an odd Poison structure on $X$, we denote the corresponding filtered deformation of $\Omega(X)$ by $\Omega_{\pi}(X)$. Since our construction is natural, for any Poisson map $X_{1} \rightarrow X_{2}$ we have a pullback map $\Omega_{\pi_{2}}\left(X_{2}\right) \rightarrow \Omega_{\pi_{1}}\left(X_{1}\right)$ preserving all the structure; hence, e.g., if $X$ is a Poisson Lie group, $\Omega_{\pi}(X)$ is a (differential filtered) Hopf algebra.

Let us give some examples of odd Poisson manifolds and of their algebras $\Omega_{\pi}(X)$. If $X=\Pi T^{*} M$ with its canonical odd symplectic form, $\Omega_{\pi}(X)$ is the algebra of differential operators acting on differential forms on $M$. The operator corresponding to a function on $X$, i.e. to a multivector field $m$ on $M$, is $i_{m}$. The differential is the supercommutator with the de Rham $d$.

As another example, if $\mathfrak{g}$ is a Lie algebra, take $X=\Pi \mathfrak{g}^{*}$ with its Kirillov-Kostant Poisson structure. Geometrically, $\Omega_{\pi}(X)$ is the convolution algebra of deRham currents on the group $G$, supported at $1 \in G$. More algebraically, it is the crossed product of $\mathfrak{A} \mathfrak{g}$ with $\bigwedge \mathfrak{g}$; the differential maps identically $\mathfrak{g} \subset \bigwedge \mathfrak{g}$ to $\mathfrak{g} \subset \mathfrak{A} \mathfrak{g}$.

These two examples were special cases of $X=\Pi A^{*}$, where $A \rightarrow M$ is a Lie algebroid. Lie theory is a source of other interesting odd Poisson structures on graded supermanifolds. Recall these important observations of A. Vaintrob [2]: A Lie algebroid structure on $A$ is equivalent to a degree -1 odd Poisson structure $\pi$ on $A^{*}[1]$ (this is the one we just mentioned) and also to a degree 1 odd vector field $Q$ on $A[1]$ with $Q^{2}=0$. A bialgebroid structure on $A$ is both $\pi$ and $Q$ on $A^{*}[1]$, such that $\mathcal{L}_{Q} \pi=0$. They can be combined to a single Poisson structure $\tilde{\pi}=\pi+Q \partial_{t}$ on $A^{*}[1] \times \mathbb{R}[2]$ (here $t$ is the coordinate on $\mathbb{R}[2]$; recall that an odd Poisson structure on $X$ is an odd quadratic function $\pi$ on $T^{*} X$ such that $\{\pi, \pi\}=0 ; Q \partial_{t}$ is understood as a product of two linear functions on $\left.T^{*}\left(\Pi A^{*} \times \mathbb{R}\right)\right)$.

A quasi-bialgebroid structure on $A$ is a graded principal $\mathbb{R}[2]$-bundle $X \rightarrow A^{*}[1]$ with a $\mathbb{R}[2]$-invariant odd Poisson structure $\tilde{\pi}$ of degree -1 . Choosing a trivialization $X=A^{*}[1] \times \mathbb{R}[2]$ we get a decomposition $\tilde{\pi}=\pi+Q \partial_{t}+\phi \partial_{t}^{2}$, where $\pi$ is a degree -1 odd Poisson structure on $A^{*}[1], Q$ a degree 1 odd vector field on $A^{*}[1]$ and $\phi$ a degree 3 odd function on $A^{*}[1](\pi, Q$ and $\phi$ satisfy the obvious equations coming from $\{\tilde{\pi}, \tilde{\pi}\}=0)$. It is not clear to me what role the differential algebra $\Omega_{\tilde{\pi}}(X)$ may play e.g. in the problem of quantization of quasi-bialgebroids. 


\section{Khudaverdian's BV operator}

In this section we recall several theorems of $\mathrm{H}$. Khudaverdian [1].

Let $Y$ be an odd symplectic manifold and let $x^{i}, \xi_{i}$ be local Darboux coordinates (i.e. $\omega=$ $\left.d x^{i} \wedge d \xi_{i}\right)$. Let

$$
\Delta=\frac{\partial^{2}}{\partial x^{i} \partial \xi_{i}},
$$

understood as a differential operator from semidensities to semidensities. Then

Theorem 2. 1. $\Delta$ is odd and $\Delta^{2}=0$

2. $\Delta$ is formally selfadjoint

3. $\mathcal{L}_{X_{f}}=[\Delta, f]$, where $X_{f}$ is the Hamiltonian vector field generated by $f$ and $[$,$] the$ supercommutator

4. $\Delta$ is independent of the choice of Darboux coordinates

Proof. This is just a sketch: 1 . and 2. are evident and 3. can be directly computed. From 1. and 3. we get that $\left[\mathcal{L}_{X_{f}}, \Delta\right]=0$, i.e. $\Delta$ is invariant under Hamiltonian diffeomorphisms, hence we get 4 .

Notice that Hamiltonian diffeomorphisms act trivially of the cohomology of $\Delta$, since by 3. they are homotopies. If $Y=\Pi T^{*} M$ then one can identify differential forms on $M$ with semidensities on $Y$ using Fourier transform along the fibres of $\Pi T M$; then $\Delta$ becomes $d$ and 3. contains as a special case Cartan formula.

If $\alpha$ and $\beta$ are semidensities on $Y$, we set

$$
(\alpha, \beta)=\int_{Y} \alpha \beta
$$

(provided the integral is well defined). Since $\Delta$ is formally selfadjoint, if $\alpha$ is $\Delta$-closed and $\beta$ is $\Delta$-exact then $(\alpha, \beta)=0$, i.e. $($,$) is well defined on cohomology classes (provided the$ appropriate care is taken for the finiteness of the integral).

Let $L \subset Y$ be a Lagrangian submanifold. We define a $\delta$-like generalized semidensity $\delta_{L}$ supported on $L$ : in local Darboux coordinates $x^{i}, \xi_{i}$, with $L$ given by the equations $\xi_{i}=0$, we set $\delta_{L}=\prod_{i} \delta\left(\xi_{i}\right)$.

Theorem 3. $\delta_{L}$ is $\Delta$-closed and independent of the choice of coordinates

Proof. Closedness is obvious and independence can be computed.

Since Hamiltonian flows generate homotopies on semidensities, we know that $\delta_{L}$ and $\delta_{L^{\prime}}$ lie in the same cohomology class whenever $L$ and $L^{\prime}$ can be connected by a Hamiltonian diffeomorphism. Hence, if $\alpha$ is closed (and appropriate integrals are finite) then $\left(\delta_{L}, \alpha\right)=$ $\left(\delta_{L^{\prime}}, \alpha\right)$; this is the geometrical basis for BV quantization. 


\section{Quantization functor}

Definition 1. The objects of the quantum odd symplectic category (QOSC) are odd symplectic manifolds and $\operatorname{Hom}\left(Y_{1}, Y_{2}\right)$ is the $\mathbb{Z}_{2}$-graded complex of generalized semidensities on $\bar{Y}_{1} \times Y_{2}\left(\bar{Y}_{1}\right.$ denotes $Y_{1}$ with the opposite sympectic form); composition of morphisms is given by integration.

Notice that the composition is not always defined, hence we don't really have a category. This is the same problem as with the symplectic category; we'll keep it in mind and ignore it.

Composition is $\Delta$-equivariant since $\Delta$ is formally selfadjoint. The quantization of an odd symplectic manifold $Y$ can be defined as $\operatorname{Hom}(p t, Y)$, i.e. as the space of generalized semidensities on $Y$.

Definition 2. The quantization functor from the odd symplectic category to QOSC is the identity on objects and it maps any Lagrangian relation $L$ to $\delta_{L}$.

Of course, we have to prove that composition of $L$ 's corresponds to composition of $\delta_{L}$ 's. This is quite simple. If two relations $L_{1}$ and $L_{2}$ can be composed (i.e. they satisfy the transversality condition) and their composition is $L$, the composition of $\delta_{L_{1}}$ with $\delta_{L_{2}}$ is a $\delta$-like distribution supported at $L$, i.e. a multiple of $\delta_{L}$. Since both $\delta_{L_{1}}$ and $\delta_{L_{2}}$ are closed, so must be their composition, i.e. it is a constant multiple of $\delta_{L}$. Finally, we have to verify that the constant is 1 . It is enough to do it at a single point. For example, we can locally deform $L_{1}$ and $L_{2}$ so that they are both given by setting some of chosen Darboux coordinates to 0 . For such $L$ 's the constant is clearly 1 , therefore it is 1 also in the undeformed parts of $L$ 's, and so it is 1 for the original $L$ 's as well.

\section{Differential forms on odd Poisson supermanifolds II}

Now the following dream is fulfilled: whenever we have an algebraic structure in the odd symplectic world, we get a corresponding structure in the world of $\mathbb{Z}_{2}$-graded chain complexes. As the most obvious example, let us start with an odd Poisson manifold $X$ and let $Y$ be its (local) odd symplectic groupoid; the quantization of $Y$ will be a differential algebra.

The quantization of $Y$ is the space of generalized semidensities on $Y$. We choose a reasonable subspace so that the product in the algebra (given by composition with $\delta_{L} \in$ $\operatorname{Hom}(Y \times Y, Y)$, where $L$ is the graph of the product in the groupoid $Y)$ is well defined. A cheap choice is to take smooth multiples of $\delta_{X}$ and of its derivatives (here $X \subset Y$ is the Lagrangian submanifold of units of the groupoid $Y$ ). In this way we get a differential algebra with an increasing filtration (given by the degree of the distributions). As one easily sees, it is the algebra $\Omega_{\pi}(X)$.

\section{References}

[1] H. Khudaverdian, Semidensities on odd symplectic supermanifolds, math.DG/0012256

[2] A. Vaintrob, Lie algebroids and homological fields, Russian Math. Surveys 52 (1997) no.2

[3] A. Weinstein, The symplectic "category". In: Differential geometric methods in mathematical physics (Clausthal 1980), 45-41. Springer, Berlin 1982. 